MATHEMATICS OF COMPUTATION

Volume 73 , Number 246 , Pages 691-717

S 0025-5718(03)01607-7

Article electronically published on November 24, 2003

\title{
SEQUENTIAL AND PARALLEL SYNCHRONOUS ALTERNATING ITERATIVE METHODS
}

\author{
JOAN-JOSEP CLIMENT, CARMEN PEREA, LEANDRO TORTOSA, \\ AND ANTONIO ZAMORA
}

\begin{abstract}
The so-called parallel multisplitting nonstationary iterative Model A was introduced by Bru, Elsner, and Neumann [Linear Algebra and its Applications 103:175-192 (1988)] for solving a nonsingular linear system $A \boldsymbol{x}=\boldsymbol{b}$ using a weak nonnegative multisplitting of the first type. In this paper new results are introduced when $A$ is a monotone matrix using a weak nonnegative multisplitting of the second type and when $A$ is a symmetric positive definite matrix using a $P$-regular multisplitting. Also, nonstationary alternating iterative methods are studied. Finally, combining Model A and alternating iterative methods, two new models of parallel multisplitting nonstationary iterations are introduced. When matrix $A$ is monotone and the multisplittings are weak nonnegative of the first or of the second type, both models lead to convergent schemes. Also, when matrix $A$ is symmetric positive definite and the multisplittings are $P$-regular, the schemes are also convergent.
\end{abstract}

\section{INTRODUCTION}

For the solution of a real linear system

$$
A \boldsymbol{x}=\boldsymbol{b},
$$

where $A$ is a given nonsingular, large, and sparse matrix, $\boldsymbol{x}$ is the unknown vector, and $\boldsymbol{b}$ is a given vector, it is customary to consider an iterative method.

The nonstationary iterative methods based on the concepts of splitting and multisplitting (see Section 2 for definitions) can be expressed as a standard iterative scheme

$$
\boldsymbol{x}^{(k+1)}=T^{(k)} \boldsymbol{x}^{(k)}+\boldsymbol{c}^{(k)}, \quad k=0,1,2, \ldots
$$

Let $\boldsymbol{\xi}$ be the exact solution of systems (1). If we define the error vector at the $k$ th iteration as $\boldsymbol{e}^{(k)}=\boldsymbol{x}^{(k)}-\boldsymbol{\xi}$, then by (2) we have that

$$
\boldsymbol{e}^{(k+1)}=T^{(k)} \boldsymbol{e}^{(k)}=T^{(k)} T^{(k-1)} \ldots T^{(1)} T^{(0)} \boldsymbol{e}^{(0)}, \quad k=0,1,2, \ldots
$$

Consequently, the convergence of the iterative process (2) to the unique solution of system (1) for any initial vector $\boldsymbol{x}^{(0)}$ is equivalent to

$$
\lim _{k \rightarrow \infty}\left(T^{(k)} T^{(k-1)} \cdots T^{(1)} T^{(0)}\right)=0,
$$

Received by the editor July 9, 2001 and, in revised form, November 13, 2002.

2000 Mathematics Subject Classification. Primary 65F10, 65F15.

Key words and phrases. Nonsingular matrix, iterative method, spectral radius, splitting, multisplitting, alternating method, stationary method, nonstationary method, convergence conditions, comparison conditions. 
which in turn is equivalent, by Theorem 2.4.2 of Young [27, to

$$
\lim _{k \rightarrow \infty}\left\|T^{(k)} T^{(k-1)} \cdots T^{(1)} T^{(0)}\right\|=0,
$$

for every matrix norm $\|\cdot\|$.

For the particular case in that $T^{(k)}=T$, for $k=0,1,2 \ldots$, we obtain the standard stationary iterative scheme

$$
\boldsymbol{x}^{(k+1)}=T \boldsymbol{x}^{(k)}+\boldsymbol{c}, \quad k=0,1,2, \ldots
$$

It is well known that the iterative process (5) converges to the unique solution of system (1) if and only if $\rho(T)<1$, where $\rho(T)$ denotes the spectral radius of the iteration matrix $T$, which is equivalent to equality (4) for $T^{(k)}=T$.

There are many well-known convergence and comparison conditions for the iterative process (5) depending on matrix $A$ and the class of the splitting or multisplitting considered. See, for example, Varga [24, Bermann and Plemmons [1], Miller and Neumann [18, Csordas and Varga 9, O'Leary and White [22, Woźnicki 26], Nabben [20, and Climent and Perea 6, 77. For convergence results for the iterative scheme (2), based on the concept of multisplitting, see, for example, Bru, Elsner and Neuman 3, 4] and Migallón, Penadés and Szyld [19.

The purpose of this paper is to introduce new sequential and parallel synchronous nonstationary alternating iterative methods. We start, in the next section, with the notation and the preliminary results. In Section 3 we introduce the main results for nonstationary sequential alternating iterative methods, as well as some results for the corresponding stationary sequential alternating iterative method. On the other hand, in Section 4 we present the parallel nonstationary Model A introduced by Bru, Elsner and Neuman [3] extending some convergence results. Next, in Section 5, combining sequential alternating iterative methods introduced in Section 3 and the parallel nonstationary Model A, we introduce two new parallel iterative methods, and we establish different convergence and comparison results for it. Finally, in Section [6] we present some numerical results.

\section{Preliminary Results}

A real $n \times n$ matrix $A=\left[a_{i j}\right]$ is called nonnegative (respectively, positive) and denoted $A \geq 0$ (respectively, $A>0$ ) if $a_{i j} \geq 0$ (respectively, $a_{i j}>0$ ) for $1 \leq i, j \leq n$. In accordance with this notation, if $A, B$ are real $n \times n$ matrices, we say that $A \geq B$ (respectively, $A>B$ ) if $A-B \geq 0$ (respectively, $A-B>0$ ) and similarly for vectors. A real matrix $A$ is called monotone if $A$ is nonsingular and $A^{-1} \geq 0$.

In the following definition we present the different types of splittings that appear in this paper (see for example Varga [24, Berman and Plemmons 1], Marek and Szyld [17, Ortega 23], Woźnicki [26], and Climent and Perea [6, 7]).

Definition 1. Let $A$ be an $n \times n$ matrix. The representation $A=M-N$ is called a splitting of $A$ if $M$ is a nonsingular matrix. In addition, the splitting is

- convergent if $\rho\left(M^{-1} N\right)<1$;

- regular if $M^{-1} \geq 0$ and $N \geq 0$;

- nonnegative if $M^{-1} \geq 0, M^{-1} N \geq 0$, and $N M^{-1} \geq 0$;

- weak nonnegative of the first type if $M^{-1} \geq 0$ and $M^{-1} N \geq 0$; weak nonnegative of the second type if $M^{-1} \geq 0$ and $N M^{-1} \geq 0$; 
- $P$-regular if $M^{T}+N$ is a positive definite matrix.

O'Leary and White 22] introduce the concept of multisplitting, as a generalization of the concept of splitting, to solve linear system (11) in parallel.

Definition 2. We say that the set of splittings $\left\{\left(M_{l}, N_{l}, E_{l}\right)\right\}_{l=1}^{p}$ is a multisplitting of $A$ if

- $A=M_{l}-N_{l}$, for $l=1,2, \ldots, p$, is a splitting,

- $E_{l} \geq 0$, for $l=1,2, \ldots, p$, are diagonal matrices called weighting matrices,

- $\sum_{l=1}^{p} E_{l}=I$ where $I$ is the identity matrix.

As a generalization of Definition 1, we say that a multisplitting is regular, nonnegative, weak nonnegative of the first type, weak nonnegative of the second type, or $P$-regular, respectively, if each splitting of the multisplitting is regular, nonnegative, weak nonnegative of the first type, weak nonnegative of the second type, or $P$-regular, respectively.

The necessity to distinguish between weak nonnegative splittings of the first type and weak nonnegative splittings of the second type is motivated by the fact that there exist convergent splittings that are weak nonnegative of the second type but not of the first type, and therefore, using the known results for weak nonnegative splittings of the first type (also called weak regular splittings for many authors), we cannot ensure the convergence of the splittings (see Climent and Perea 6, Example 2]).

For practical reasons, for the splitting $A=M-N$ it is assumed that $M$ is a matrix for which efficient solution methods are known to exist. However, in some practical problems arising in many areas of science and engineering, the matrices are large-sized and sparse except in a few rows or columns that are dense. For matrices with a few dense columns a natural splitting usually is of the first type and for the matrices with a few dense rows a natural splitting usually is of the second type.

Lanzkron, Rose, and Szyld [15, Lemma 2.3] introduce the first part of the following result which is very useful in studying alternating iterative methods (see also Benzi and Szyld [2]) when considering nonnegative splittings of the first type. In a similar way, we introduce the second part of the following result to be used when considering nonnegative splittings of the second type.

Lemma 1. Let $A$ and $R$ be square matrices such that $A$ and $I-R$ are nonsingular. Then

(i) there exists a unique pair of matrices $B_{1}$ and $C_{1}$, such that $B_{1}$ is nonsingular, $R=B_{1}^{-1} C_{1}$ and $A=B_{1}-C_{1}$; the matrices are $B_{1}=A(I-R)^{-1}$ and $C_{1}=B_{1}-A$;

(ii) there exists a unique pair of matrices $B_{2}$ and $C_{2}$, such that $B_{2}$ is nonsingular, $R=C_{2} B_{2}^{-1}$ and $A=B_{2}-C_{2}$; the matrices are $B_{2}=(I-R)^{-1} A$ and $C_{2}=B_{2}-A$.

According with Lemma 1 we say that matrix $R$ induces a unique splitting $A=$ $B_{1}-C_{1}$ such that $R=B_{1}^{-1} C_{1}$ and a unique splitting $A=B_{2}-C_{2}$ such that $R=C_{2} B_{2}^{-1}$. Observe that both splittings are different unless matrices $A$ and $(I-R)^{-1}$ commute. 
As we will see in the next sections, Lemma 1 is fundamental in obtaining the simplest way of tackling the question of convergence of different stationary iterative methods. Moreover, under some hypotheses that we present in the following result, we can establish an important relation between the splittings obtained in the above lemma.

Lemma 2. Let $A$ and $R$ be square matrices such that $A$ and $I-R$ are nonsingular. If $A=B_{1}-C_{1}$ is the unique splitting induced by matrix $T$ such that $T=B_{1}^{-1} C_{1}$ and if $A=B_{2}-C_{2}$ is the unique splitting induced by matrix $A T A^{-1}$ such that $A T A^{-1}=C_{2} B_{2}^{-1}$, then $B_{1}=B_{2}$ and $C_{1}=C_{2}$.

Proof. By Lemma 1 taking into account the definition of $B_{1}$ and $B_{2}$.

\section{Sequential alternating iterative methods}

Consider the general class of alternating iterative methods for the solution of system (11) of the form

$$
\begin{gathered}
\boldsymbol{x}^{(k+j / q)}=\left(M_{j}^{-1} N_{j}\right)^{\mu(j, k)} \boldsymbol{x}^{(k+(j-1) / q)}+\sum_{i=0}^{\mu(j, k)-1}\left(M_{j}^{-1} N_{j}\right)^{i} M_{j}^{-1} \boldsymbol{b}, \\
j=1,2, \ldots, q, \quad k=0,1,2, \ldots,
\end{gathered}
$$

where

$$
A=M_{j}-N_{j}, \quad j=1,2, \ldots, q,
$$

is a splitting of $A, \boldsymbol{x}^{(0)}$ is the initial guess, and $\mu(j, k)$ is the number of iterations with the $j$ th splitting at the $k$ th global iteration.

There are different methods belonging to this class; see for example Young [27], Conrad and Wallach [8, Marchuk 16], and Benzi and Szyld 2] for the case $p=2$ and $\mu(1, k)=\mu(2, k)=1$ for $k=0,1,2, \ldots$

To analyze the converge of the alternating iterative method (6), we can eliminate vectors $\boldsymbol{x}^{(k+j / q)}$, for $j=1,2, \ldots, q$, obtaining in this way the single iterative process

$$
\begin{aligned}
& \boldsymbol{x}^{(k+1)}=\prod_{j=1}^{q}\left(M_{q-j+1}^{-1} N_{q-j+1}\right)^{\mu(q-j+1, k)} \boldsymbol{x}^{(k)} \\
& +\sum_{i=1}^{q}\left[\prod_{j=1}^{q-i}\left(M_{q-j+1}^{-1} N_{q-j+1}\right)^{\mu(q-j+1, k)}\right]\left[\sum_{l=0}^{\mu(i, k)-1}\left(M_{i}^{-1} N_{i}\right)^{l} M_{i}^{-1} \boldsymbol{b}\right] \\
& k=0,1,2, \ldots
\end{aligned}
$$

where we assume that $\prod_{j=1}^{0}\left(M_{q-j+1}^{-1} N_{q-j+1}\right)^{\mu(q-j+1, k)}=I$, which is of the form (2), with

$$
T^{(k)}=\prod_{j=1}^{q}\left(M_{q-j+1}^{-1} N_{q-j+1}\right)^{\mu(q-j+1, k)}, \quad k=0,1,2, \ldots
$$

Before we establish convergence results for the nonstationary iterative method (8), we introduce the following technical result. 
Lemma 3. For $k=0,1,2, \ldots$, let $G_{k}$ be a nonnegative matrix. Let $P$ be a nonsingular matrix, and consider matrix

$$
F_{k}=P^{-1} G_{k} P, \quad k=0,1,2, \ldots
$$

Assume also that there exists a real number $0 \leq \beta<1$ and a vector $\boldsymbol{x}>0$ such that

$$
G_{k} \boldsymbol{x} \leq \beta \boldsymbol{x}, \quad k=0,1,2, \ldots
$$

Then

$$
\lim _{k \rightarrow \infty}\left(G_{k} G_{k-1} \cdots G_{1} G_{0}\right)=\lim _{k \rightarrow \infty}\left(F_{k} F_{k-1} \cdots F_{1} F_{0}\right)=0 .
$$

Proof. From (11) it is easy to see that

$$
G_{k} G_{k-1} \cdots G_{1} G_{0} \boldsymbol{x} \leq \beta^{k} \boldsymbol{x}
$$

and using the fact that $G_{k} \geq 0$ and that $\boldsymbol{x}>0$, we obtain

$$
0 \leq \lim _{k \rightarrow \infty} G_{k} G_{k-1} \cdots G_{1} G_{0} \boldsymbol{x} \leq \lim _{k \rightarrow \infty} \beta^{k} \boldsymbol{x}=0
$$

and consequently $\lim _{k \rightarrow \infty}\left(G_{k} G_{k-1} \cdots G_{1} G_{0}\right)=0$.

Now, taking into account (10), we have that $\lim _{k \rightarrow \infty}\left(F_{k} F_{k-1} \cdots F_{1} F_{0}\right)=0$.

Next, we can establish the convergence of the nonstationary iterative process (8).

Theorem 1. Let $A$ be a nonsingular and monotone matrix. Assume that splittings (7) are weak nonnegative of the same type. Then the nonstationary iterative method (8) converges for any initial vector $\boldsymbol{x}^{(0)}$ to the unique solution of system (1).

Proof. Suppose that the splittings (7) are weak nonnegative of the first type. Then from (9) we have that $T^{(k)} \geq 0$ for $k=0,1,2, \ldots$.

Since $A^{-1} \geq 0$, there exists a vector $\boldsymbol{x}>0$ (e.g. $\left.\boldsymbol{x}=A^{-1}(1,1, \ldots, 1)^{T}\right)$ such that $A \boldsymbol{x}>0$. So

$$
\boldsymbol{x}-M_{j}^{-1} N_{j} \boldsymbol{x}=M_{j}^{-1} A \boldsymbol{x}>0
$$

and for some suitable constants $0 \leq \beta_{j}<1$ we have that

$$
M_{j}^{-1} N_{j} \boldsymbol{x} \leq \beta_{j} \boldsymbol{x}, \quad j=1,2, \ldots, q .
$$

Now, if $\beta=\max _{1 \leq j \leq q}\left\{\beta_{j}\right\}$, then from (91) it is easy to see that

$$
T^{(k)} \boldsymbol{x} \leq \beta \boldsymbol{x}, \quad k=0,1,2, \ldots,
$$

and by Lemma 3, we obtain that

$$
\lim _{k \rightarrow \infty}\left(T^{(k)} T^{(k-1)} \cdots T^{(1)} T^{(0)}\right)=0 .
$$

So, the nonstationary iterative process (즈) converges to the unique solution of system (1). Then

Now, suppose that the splittings (7) are weak nonnegative of the second type.

$$
S^{(k)}=\prod_{j=1}^{q}\left(N_{q-j+1} M_{q-j+1}^{-1}\right)^{\mu(q-j+1, k)} \geq 0, \quad k=0,1,2, \ldots
$$

Since $A^{-1} \geq 0$, there exists a vector $\boldsymbol{y}>0$ (e.g. $\left.\boldsymbol{y}^{T}=(1,1, \ldots, 1) A^{-1}\right)$ such that $\boldsymbol{y}^{T} A>0$. Then

$$
\boldsymbol{y}^{T}-\boldsymbol{y}^{T} N_{j} M_{j}^{-1}=\boldsymbol{y}^{T} A M_{j}^{-1}>0
$$


and for some suitable constants $0 \leq \gamma_{j}<1$ we have that

$$
\boldsymbol{y}^{T} N_{j} M_{j}^{-1} \leq \gamma_{j} \boldsymbol{y}^{T}, \quad j=1,2, \ldots, q .
$$

If $\gamma=\max _{1 \leq j \leq q}\left\{\gamma_{q}\right\}$, then it is easy to see that

$$
\boldsymbol{y}^{T} S^{(k)} \leq \gamma \boldsymbol{y}^{T}, \quad k=0,1,2, \ldots
$$

Now, by Theorem 3.1 of Woźnicki [26] we have that

$$
M_{j}^{-1} N_{j} A^{-1}=A^{-1} N_{j} M_{j}^{-1}, \quad j=1,2, \ldots, q,
$$

and therefore $T^{(k)}=A^{-1} S^{(k)} A$. So, equality (12) holds by Lemma [3, and the nonstationary iterative method (8) converges to the unique solution of system (1).

We remark that the number of iterations on each one of the splittings not only depends of the splitting, but it also depends of the index of the present iteration. But, if in the nonstationary process (8) we consider the particular case $\mu(j, k)=\mu_{j}$, for $k=0,1,2, \ldots$, that is, the number of iterations on each splitting depends only of the splitting, then we obtain the stationary version of the iterative process (8) which is of the form (5) with

$$
T=\prod_{j=1}^{q}\left(M_{q-j+1}^{-1} N_{q-j+1}\right)^{\mu_{q-j+1}}
$$

and a certain vector $\boldsymbol{c}$. For this stationary method we introduce the following results.

Theorem 2. Let $A$ be a nonsingular and monotone matrix. Assume that splittings (7) are weak nonnegative of the first (respectively, second) type. If $\mu(j, k)=\mu_{j}$, for $k=0,1,2, \ldots$, then the stationary version of the iterative method 8) is convergent. Furthermore, the unique splitting $A=B-C$ induced by matrix $T$ in (13) such that $T=B^{-1} C$ is also weak nonnegative of the first (respectively, second) type.

Proof. By Theorem 1 the stationary version of the iterative method (8) is convergent; that is, $\rho(T)<1$ and consequently matrix $I-T$ is nonsingular.

Suppose that the splittings (7) are weak nonnegative of the first type, and by Lemma 1 let $A=B-C$ be the unique splitting induced by matrix $T$ such that $T=B^{-1} C$. Then, from (13) clearly $B^{-1} C \geq 0$.

On the other hand, by Lemma 1 and some algebraic manipulation, we obtain that

$$
\begin{aligned}
B^{-1} & =(I-T) A^{-1} \\
& =\sum_{j=1}^{q}\left[\prod_{l=j}^{q-1}\left(M_{q-l+j}^{-1} N_{q-l+j}\right)^{\mu_{q-l+j}}\right]\left[\sum_{i=0}^{\mu_{j}-1}\left(M_{j}^{-1} N_{j}\right)^{i}\right] M_{j}^{-1}
\end{aligned}
$$

where we assume that $\prod_{l=q}^{q-1}\left(M_{2 q-l}^{-1} N_{2 q-l}\right)^{\mu_{2 q-l}}=I$. So, $A=B-C$ is a weak nonnegative splitting of the first type. 
Now, assume that the splittings (7) are weak nonnegative of the second type. Clearly

$$
S=\prod_{j=1}^{q}\left(N_{q-j+1} M_{q-j+1}^{-1}\right)^{\mu_{q-j+1}} \geq 0
$$

and after some algebraic manipulation

$$
\begin{aligned}
& A^{-1}(I-S) \\
& \quad=\sum_{j=1}^{q} M_{j}^{-1}\left[\sum_{i=0}^{\mu_{j}-1}\left(N_{j} M_{j}^{-1}\right)^{i}\right]\left[\prod_{l=1}^{j-1}\left(N_{j-l} M_{j-l}^{-1}\right)^{\mu_{j-l}}\right] \geq 0
\end{aligned}
$$

where we assume that $\prod_{l=1}^{0}\left(N_{1-l} M_{1-l}^{-1}\right)^{\mu_{1-l}}=I$.

Now, as in Theorem 11 taking into account Theorem 3.1 of Woźnicki [26], it is easy to see that $S=A T A^{-1}$, and therefore $\rho(S)<1$; that is, matrix $I-S$ is nonsingular. Then, by Lemma2, the unique splitting $A=B-C$ induced by matrix $T$ such that $T=B^{-1} C$ also satisfies $C B^{-1}=S \geq 0$ from (15), and

$$
B^{-1}=A^{-1}(I-S) \geq 0
$$

from (16). So, $A=B-C$ is a weak nonnegative splitting of the second type.

For a monotone matrix $A$, Theorem 2 establishes the convergence of the stationary version of the iterative process (8) when the splittings (7) are weak nonnegative of the first type or weak nonnegative of the second type. However, if not all the splittings in (7) are weak nonnegative of the same type, then we cannot guarantee the convergence of the stationary version of the iterative process (8) as we can see in the following example.

Example 1. Consider the monotone matrix

$$
A=\left[\begin{array}{rrr}
1 & -1 & 0 \\
-1 & 2 & 0 \\
0 & 0 & 1
\end{array}\right]
$$

and the splittings $A=M_{1}-N_{1}=M_{2}-N_{2}$, where

$$
M_{1}=\left[\begin{array}{rrr}
1 & 0 & 0 \\
-1 & 1 & 0 \\
0 & 0 & 1
\end{array}\right] \quad \text { and } \quad M_{2}=\left[\begin{array}{rrr}
1 & -1 & 0 \\
0 & 1 & 0 \\
0 & 0 & 1
\end{array}\right] \text {. }
$$

It is easy to see that the splitting $A=M_{1}-N_{1}$ is weak nonnegative of the first type but not of the second type and that the splitting $A=M_{2}-N_{2}$ is weak nonnegative of the second type but not of the first type. Now, since

$$
M_{2}^{-1} N_{2} M_{1}^{-1} N_{1}=\left[\begin{array}{ccc}
0 & 1 & 0 \\
0 & 1 & 0 \\
0 & 0 & 0
\end{array}\right]
$$

the stationary version of the iterative process (8) does not converge.

Observe that if we take $q=2$, we assume that the splittings are weak nonnegative of the first type, and we consider $\mu_{1}=\mu_{2}=1$. Then we obtain Theorem 3.2 of Benzi and Szyld [2] as a corollary of Theorem 2] 
On the other hand, as Benzi and Szyld [2, Example 3.1] show, the convergence of the individual splittings in (7) does not guarantee the convergence of the iterative scheme (15) for matrix $T$ defined by (13); that is, the stationary version of the iterative method (8), and as a consequence, although we have the convergence of the iterative process, we cannot ensure that it converges faster than some or all of the iterative process corresponding with the basic splittings. However, in the following results we introduce an upper bound for the spectral radius of the iteration matrix $T$ defined by (13). The proof of the first part is similar to the proof of Theorem 4.1 of Benzi and Szyld [2] using Theorem 3.7 of Woźnicki [26] instead of the lemma of Elsner [10].

Theorem 3. Let $A$ be a nonsingular and monotone matrix. Consider the $q$ splittings defined by (71) and matrix $T$ defined by (13). Assume also that $\mu(j, k)=\mu_{j}$ for $k=0,1,2, \ldots$.

(i) If the splittings are weak nonnegative of the first or of the second type, and $A=M_{1}-N_{1}$ is nonnegative, then

$$
\rho(T) \leq \rho\left[\left(M_{1}^{-1} N_{1}\right)^{\mu_{1}}\right] .
$$

(ii) If the splittings are weak nonnegative of the first or of the second type, and $A=M_{q}-N_{q}$ is nonnegative, then

$$
\rho(T) \leq \rho\left[\left(M_{q}^{-1} N_{q}\right)^{\mu_{q}}\right] .
$$

Proof. (i) Assume that the splittings are weak nonnegative of the first (respectively, second) type. Consider matrices $T_{1}=\left(M_{1}^{-1} N_{1}\right)^{\mu_{1}}$ and

$$
T_{2}=\prod_{j=1}^{q-1}\left(N_{q-j+1} M_{q-j+1}^{-1}\right)^{\mu_{q-j+1}} .
$$

Since $\rho\left(T_{1}\right)<1$, by Theorem 2 the unique splitting $A=B_{1}-C_{1}$ induced by matrix $T_{1}$ such that $T_{1}=B_{1}^{-1} C_{1}$ is nonnegative. Similarly, the unique splitting $A=B_{2}-C_{2}$ induced by matrix $T_{2}$ such that $T_{2}=B_{2}^{-1} C_{2}$ is weak nonnegative of the first (respectively, second) type.

Clearly $T=T_{2} T_{1}$ and $\rho(T)<1$, and by Theorem 2 the unique splitting $A=$ $B-C$ induced by matrix $T$ such that $T=B^{-1} C$ is weak nonnegative of the first (respectively, second) type. So, we can consider that $A=B-C=B_{1}-C_{1}$ are weak nonnegative splittings of different type.

Now, by Lemma 1 it is easy to see that

$$
B^{-1}=B_{1}^{-1}+B_{2}^{-1} C_{1} B_{1}^{-1} \geq B_{1}^{-1}
$$

and by Theorem 3.7 of Woźnicki [26] (see also Climent and Perea [6, Theorem 7] for a more general case), we have that

$$
\rho\left(B^{-1} C\right) \leq \rho\left(B^{-1} C_{1}\right)
$$

and inequality (18) holds.

(ii) Similar to part (i) considering

$$
T_{1}=\prod_{j=2}^{q}\left(N_{q-j+1} M_{q-j+1}^{-1}\right)^{\mu_{q-j+1}}
$$

and $T_{2}=\left(M_{q}^{-1} N_{q}\right)^{\mu_{q}}$ and taking into account inequality

$$
B^{-1}=B_{2}^{-1}+B_{1}^{-1} C_{2} B_{2}^{-1} \geq B_{2}^{-1}
$$

instead of (19). 
Finally, applying recursively the above theorem, we obtain the following result that was introduced by Benzi and Szyld [2, Theorem 4.1] for two regular splittings $A=M_{1}-N_{1}=M_{2}-N_{2}$.

Corollary 1. Let $A$ be a nonsingular and monotone matrix. If the splittings (7) are nonnegative and $\mu(k, j)=\mu_{j}$ for $k=0,1,2, \ldots$, then the following upper bound for the spectral radius of the iteration matrix $T$ defined by (13) holds:

$$
\rho(T) \leq \min _{1 \leq j \leq q}\left\{\rho\left[\left(M_{j}^{-1} N_{j}\right)^{\mu_{j}}\right]\right\} .
$$

Now, using the matrix norm induced by a symmetric positive definite matrix, we introduce the following result for the nonstationary iterative method (8) when the splittings (7) are $P$-regular.

Theorem 4. Let $A$ be a symmetric positive definite matrix. Assume that splittings (77) are P-regular. Then the nonstationary iterative method (8) converges for any initial vector $\boldsymbol{x}^{(0)}$ to the unique solution of system (1).

Proof. Since the splittings (77) are $P$-regular, by Theorem 2.4 of Frommer and Szyld [13] there exist constants $0 \leq \beta_{j}<1$ such that

$$
\left\|M_{j}^{-1} N_{j}\right\|_{A} \leq \beta_{j}, \quad j=1,2, \ldots, q .
$$

Now, if $\beta=\max _{1 \leq j \leq q}\left\{\beta_{j}\right\}$, then from (9) and the properties of matrix norms it is easy to see that

$$
\left\|T^{(k)}\right\|_{A} \leq \beta, \quad k=0,1,2, \ldots,
$$

and consequently, from (3) we obtain that

$$
\lim _{k \rightarrow \infty}\left\|T^{(k)} T^{(k-1)} \cdots T^{(1)} T^{(0)}\right\|_{A} \leq \lim _{k \rightarrow \infty} \beta^{k}=0
$$

and the nonstationary iterative process $(8)$ is convergent.

Moreover, if we consider the particular case $\mu(j, k)=\mu_{j}$, for $k=0,1,2, \ldots$, then we obtain the following result for the stationary version of the iterative process (즈).

Theorem 5. Let $A$ be a symmetric positive definite matrix. Assume that splittings (7) are P-regular. If $\mu(j, k)=\mu_{j}$, for $k=0,1,2, \ldots$, then the stationary version of the iterative method (8) converges to the unique solution of system (11). Furthermore, the unique splitting $A=B-C$ induced by matrix $T$ in (13) such that $T=B^{-1} C$ is also P-regular.

Proof. By Theorem 4 the stationary version of the iterative method (8) is convergent, that is, $\rho(T)<1$, and consequently matrix $I-T$ is nonsingular.

Again as in Theorem 4 by Theorem 2.4 of Frommer and Szyld [13], for a suitable constant $0 \leq \beta<1$ we have that

$$
\left\|M_{j}^{-1} N_{j}\right\|_{A}<\beta, \quad j=1,2, \ldots, q,
$$

and using the properties of matrix norms, from (13) we obtain that

$$
\|T\|_{A} \leq \prod_{j=1}^{q}\left\|M_{q-j+1}^{-1} N_{q-j+1}\right\|_{A}^{\mu_{q-j+1}}<\beta .
$$

Consequently, by Lemma 1 and Theorem 2.4 of Frommer and Szyld [13], the unique splitting $A=B-C$ induced by matrix $T$ such that $T=B^{-1} C$ is $P$-regular. 
Observe that if we take $q=2$, we consider $\mu_{1}=\mu_{2}=1$, and we assume that the splittings are $P$-regular; then we obtain Theorem 3.5 of Benzi and Szyld [2] as a corollary of Theorem 5

\section{PARAllel SynChronous iterative methods}

With the development of parallel computation in the last decades, the utilization of parallel algorithms for the solution of a large and sparse nonsingular linear system has become effective. O'Leary and White 22] introduce the concept of multisplitting (see Definition 2]) for the parallel solution of linear system (1). Let $\left\{\left(M_{l}, N_{l}, E_{l}\right)\right\}_{l=1}^{p}$ be a multisplitting and suppose that we have a multiprocessor with $p$ processors connected to a host processor, that is, the same number of processors as splittings, and that all processors have the last update vector $\boldsymbol{x}^{(k)}$; then the $l$ th processor only computes those entries of the vector

$$
M_{l}^{-1} N_{l} \boldsymbol{x}^{(k)}+M_{l}^{-1} \boldsymbol{b}
$$

which correspond to the nonzero diagonal entries of $E_{l}$. The processor then scales these entries so as to be able to deliver the vector

$$
E_{l}\left(M_{l}^{-1} N_{l} \boldsymbol{x}^{(k)}+M_{l}^{-1} \boldsymbol{b}\right)
$$

to a host processor, performing the parallel multisplitting scheme

$$
\boldsymbol{x}^{(k+1)}=\sum_{l=1}^{p} E_{l} M_{l}^{-1} N_{l} \boldsymbol{x}^{(k)}+\sum_{l=1}^{p} E_{l} M_{l}^{-1} \boldsymbol{b}, \quad k=0,1,2, \ldots
$$

Many authors, such as Neumann and Plemmons 21], Elsner [10], Frommer and Mayer [11, 12], Wang [25, Bru, Migallón, and Penadés [5], Nabben [20], introduce convergence conditions for different parallel algorithms based on the standard parallel iterative scheme (20) for different types of matrices and multisplittings.

The parallel iterative process (20) implicitly assumes that processor $l$ has to be synchronized between the formation of iterates $\boldsymbol{x}^{(k)}$ and $\boldsymbol{x}^{(k+1)}$. Moreover, in practice, it is quite usual to consider the diagonal matrices $E_{l} \geq 0$ for $l=1, \ldots, p$, with zero or one entries; then in this case in each processor we must solve some subproblems (a system of size less than $n$ ) that may be of different sizes.

Nevertheless, Bru, Elsner, and Neumann [3, with the aim of avoiding loss of time and efficiency in processor use, establish the so-called Model A.

Model A: Each processor can carry out a varying number of local iterations until a mutual phase time is reached when all the processors are ready to contribute toward the global iteration.

Consider a multisplitting of $A$,

$$
\left\{\left(M_{l}, N_{l}, E_{l}\right)\right\}_{l=1}^{p} .
$$

Bru, Elsner, and Neumann [3] establish the mathematical formulation of Model A, starting with an arbitrary vector $\boldsymbol{x}^{(0)}$, as

$$
\begin{aligned}
\boldsymbol{x}^{(k+1)}= & \sum_{l=1}^{p} E_{l}\left(M_{l}^{-1} N_{l}\right)^{\mu(k, l)} \boldsymbol{x}^{(k)} \\
& +\sum_{l=1}^{p} E_{l}\left(\sum_{i=0}^{\mu(k, l)-1}\left(M_{l}^{-1} N_{l}\right)^{i}\right) M_{l}^{-1} \boldsymbol{b},
\end{aligned}
$$


where $\mu(k, l) \geq 0$ denotes the number of local iterations in $l$ th processor for $l=$ $1,2, \ldots, p$ at the $k$ th global iteration for $k=0,1,2, \ldots$. Observe that the above parallel iterative method has the form (2) with

$$
T^{(k)}=\sum_{l=1}^{p} E_{l}\left(M_{l}^{-1} N_{l}\right)^{\mu(k, l)} .
$$

Bru, Elsner, and Neumann [3] also introduce the following convergence result for the parallel iterative method (22) that we quote for further references.

Theorem 6 (Theorem 2.1 of [3]). Let $A$ be a nonsingular and monotone matrix. Assume that the multisplitting (21) is weak nonnegative of the first type. If

$$
\mu(k, l) \geq 1, \quad l=1,2, \ldots, p, \quad k=0,1,2, \ldots,
$$

then the parallel iterative method (22) converges for any initial vector $\boldsymbol{x}^{(0)}$ to the unique solution of system (1).

If in Theorem [6 we replace "first type" with "second type" and introduce the additional hypothesis " $A E_{l}=E_{l} A$ for $l=1,2, \ldots, p$ ", we obtain the following convergence result.

Theorem 7. Let $A$ be a nonsingular and monotone matrix. Assume that the multisplitting (21) is weak nonnegative of the second type and $A E_{l}=E_{l} A$ for $l=1,2, \ldots, p$. If (24) holds, then the parallel iterative method (22) converges for any initial vector $\boldsymbol{x}^{(0)}$ to the unique solution of system (1).

Proof. Since the multisplitting is weak nonnegative of the second type, we have that

$$
S^{(k)}=\sum_{l=1}^{p} E_{l}\left(N_{l} M_{l}^{-1}\right)^{\mu(k, l)} \geq 0, \quad k=0,1,2, \ldots,
$$

and by an argument similar to that in Theorem 1 we have that $\boldsymbol{x}^{T} S^{(k)} \leq \gamma \boldsymbol{x}^{T}$ for a suitable constant $0 \leq \gamma<1$ and a vector $\boldsymbol{x}>0$.

Now, as in Theorem 1, taking into account Theorem 3.1 of Woźnicki [26], and using the fact that $A E_{l}=E_{l} A$ for $l=1,2, \ldots, p$, we obtain that $A^{-1} S^{(k)} A=T^{(k)}$ for $k=0,1,2, \ldots$, where matrix $T^{(k)}$ is defined by (23).

Then, by Lemma 3, $\lim _{k \rightarrow \infty}\left(T^{(k)} T^{(k-1)} \cdots T^{(1)} T^{(0)}\right)=0$ and therefore the iterative method (22) converges for any initial vector $\boldsymbol{x}^{(0)}$ to the unique solution of system (1).

Now if we consider a symmetric positive definite matrix $A$ and a $P$-regular multisplitting with the additional hypothesis " $E_{l}=\alpha_{l} I$ for $l=1,2, \ldots, p$ ", then we can establish the following convergence result for the parallel iterative method (22).

Theorem 8. Let $A$ be a symmetric positive definite matrix. Assume that the multisplitting (21) is P-regular and that $E_{l}=\alpha_{l} I$ for $l=1,2, \ldots, p$. If (24) holds, then the iterative method (22) converges for any initial vector $\boldsymbol{x}^{(0)}$ to the unique solution of system (1).

Proof. As in the proof of Theorem 4 there exists a suitable constant $0 \leq \beta<1$ such that

$$
\left\|M_{l}^{-1} N_{l}\right\|_{A} \leq \beta, \quad l=1,2, \ldots, p,
$$


and consequently

$$
\left\|T^{(k)}\right\|_{A} \leq \sum_{l=1}^{p} \alpha_{l}\left\|M_{l}^{-1} N_{l}\right\|_{A}^{\mu(k, l)} \leq \sum_{l=1}^{p} \alpha_{l} \beta=\beta, \quad k=0,1,2, \ldots,
$$

where matrix $T^{(k)}$ is defined in (23), and the proof follows as in Theorem4

Note that as Bru, Elsner, and Neumann [3] remark for Theorem [6, also for Theorems 7 and 8 , assumption (24) can be weakened as follows:

$$
\mu(k, l) \geq 0, \quad l=1,2, \ldots, p, \quad k=1,2, \ldots,
$$

and for infinitely many $k$ 's

$$
\mu(k, l) \geq 1, \quad l=1,2, \ldots, p .
$$

The difference between condition (24) and conditions (25)-(26) is that conditions (25) -(26) permit, if necessary, any processor to skip its condition to any major step of the iteration provided that infinitely often all processors contribute simultaneously toward a global iteration.

Recently, Migallón, Penadés, and Szyld [19] introduce a similar result to Theorem 8 when the additional hypothesis " $E_{l}=\alpha_{l} I$ " was changed by a condition on the number of local iterations $\mu(k, l)$.

Theorem 9 (Corollary 2.3 of 19 ). Let $A$ be a symmetric positive definite matrix and assume that the multisplitting (21) is P-regular. Given a fixed positive number $\theta<1$, let $\eta=\theta /\left(\sum_{l=1}^{p}\left\|E_{l}\right\|_{A}\right)$. Let $\tilde{\nu}$ be such that

$$
\left\|\left(M_{l}^{-1} N_{l}\right)^{\tilde{\nu}}\right\|_{A} \leq \eta, \quad \nu \geq \tilde{\nu}, \quad l=1,2, \ldots, p .
$$

If the sequence of the number of iterations satisfies

$$
\mu(k, l) \geq \tilde{\nu}, \quad l=1,2, \ldots, p, \quad k=0,1,2, \ldots,
$$

then the iterative method (22) converges for any initial vector $\boldsymbol{x}^{(0)}$ to the unique solution of system (1).

In a similar way, using Theorem 2.1 of Migallón, Penadés, and Szyld [19], we can change the additional hypothesis " $A E_{l}=E_{l} A$ for $l=1,2, \ldots, p$ " in Theorem 7 for a condition on the number of local iterations $\mu(k, l)$ obtaining the following result. Here, $\|\cdot\|$ denotes any matrix norm such that $\|I\|=1$.

Theorem 10. Let $A$ be a nonsingular and monotone matrix and assume that the multisplitting (21) is weak nonnegative of the second type. Given a fixed positive number $\theta<1$, let $\eta=\theta /\left(\sum_{l=1}^{p}\left\|E_{l}\right\|\right)$. Let $\tilde{\nu}$ be such that

$$
\left\|\left(M_{l}^{-1} N_{l}\right)^{\tilde{\nu}}\right\| \leq \eta, \quad \nu \geq \tilde{\nu}, \quad l=1,2, \ldots, p .
$$

If the sequence of the number of iterations satisfies inequality (27), then the iterative method (22) converges for any initial vector $\boldsymbol{x}^{(0)}$ to the unique solution of system (11). 
If in the nonstationary parallel iterative method (22) we consider the particular case in which the number of local iterations only depends of the $l$ th processor, that is, $\mu(k, l)=\mu_{l}$ for $k=0,1,2, \ldots$, then we obtain a stationary version of that method whose iteration matrix is

$$
T=\sum_{l=1}^{p} E_{l}\left(M_{l}^{-1} N_{l}\right)^{\mu_{l}}
$$

Consider also matrix

$$
R=\sum_{l=1}^{p} E_{l}\left(\sum_{i=0}^{\mu_{l}-1}\left(M_{l}^{-1} N_{l}\right)^{i}\right) M_{l}^{-1} .
$$

Now, with the purpose of introducing similar results to Theorems 2 and 5 for the stationary version of the parallel iterative method (22), we introduce the following lemma. The proof of the first part can be found in Elsner [10, the second part in Climent and Perea [7], and the last part in Nabben [20].

Lemma 4. Let $A$ be a nonsingular matrix. Let $\left\{\left(M_{l}, N_{l}, E_{l}\right)\right\}_{l=1}^{p}$ be a multisplitting of $A$ and consider matrices

$$
H=\sum_{l=1}^{p} E_{l} M_{l}^{-1} N_{l} \quad \text { and } \quad G=\sum_{l=1}^{p} E_{l} M_{l}^{-1} .
$$

(i) If $A$ is monotone and the multisplitting is weak nonnegative of the first type, then $G$ is nonsingular and the splitting $A=G^{-1}-\left(G^{-1}-A\right)$ associated with the multisplitting is also weak nonnegative of the first type.

(ii) If $A$ is monotone, the multisplitting is weak nonnegative of the second type, and $A E_{l}=E_{l} A$ for $l=1,2, \ldots, p$, then $G$ is nonsingular and the splitting $A=G^{-1}-\left(G^{-1}-A\right)$ associated with the multisplitting is also weak nonnegative of the second type.

(iii) If $A$ is symmetric positive definite, the multisplitting is $P$-regular, and $E_{l}=\alpha_{l} I$ for $l=1,2, \ldots, p$, then $G$ is nonsingular and the splitting $A=$ $G^{-1}-\left(G^{-1}-A\right)$ associated with the multisplitting is also P-regular.

As Elsner [10, Climent and Perea 7], and Nabben [20] show, it is easy to see that $H=G\left(G^{-1}-A\right)$ for matrices $H$ and $G$ of Lemma 4, So, $A=G^{-1}-\left(G^{-1}-A\right)$ is the unique splitting induced by matrix $H$ satisfying the above equality in the sense of Lemma 111i).

Finally, using the above lemma, we introduce the following results for the stationary version of the parallel iterative process (22).

Theorem 11. Let $A$ be a nonsingular matrix. Let $\left\{\left(M_{l}, N_{l}, E_{l}\right)\right\}_{l=1}^{p}$ be a multisplitting. Assume that $\mu(k, l)=\mu_{l} \geq 1$ for $k=0,1,2, \ldots$, and consider matrices $T$ and $R$ defined by (28) and (29), respectively.

(i) If $A$ is monotone and the multisplitting is weak nonnegative of the first type, then the stationary version of the parallel iterative method (22) is convergent. Moreover, matrix $R$ is nonsingular and $A=R^{-1}-\left(R^{-1}-A\right)$ is the unique weak nonnegative splitting of the first type induced by matrix $T$ such that $T=R\left(R^{-1}-A\right)$.

(ii) If $A$ is monotone, the multisplitting is weak nonnegative of the second type, and $A E_{l}=E_{l} A$ for $l=1,2, \ldots, p$, then the stationary version of the parallel iterative method (22) is convergent. Moreover, matrix $R$ is 
nonsingular and $A=R^{-1}-\left(R^{-1}-A\right)$ is the unique weak nonnegative splitting of the second type induced by matrix $T$ such that $T=R\left(R^{-1}-A\right)$.

(iii) If $A$ is symmetric positive definite, the multisplitting is $P$-regular, and $E_{l}=\alpha_{l} I$ for $l=1,2, \ldots, p$, then the stationary version of the parallel iterative method (22) is convergent. Moreover, matrix $R$ is nonsingular and $A=R^{-1}-\left(R^{-1}-A\right)$ is the unique P-regular splitting induced by matrix $T$ such that $T=R\left(R^{-1}-A\right)$.

Proof. (i) By Theorem [6, the stationary version of the parallel iterative method (22) is convergent.

Since $A$ is monotone and for $l=1,2, \ldots, p$, the splitting $A=M_{l}-N_{l}$ is weak nonnegative of the first type, by Theorem 7.5.6 of Berman and Plemmons 1] (see also Climent and Perea [6, Theorem 3]), we have that $\rho\left(M_{l}^{-1} N_{l}\right)<1$. Next, using the fact that $M_{l}^{-1} N_{l} \geq 0$, we obtain that $\rho\left[\left(M_{l}^{-1} N_{l}\right)^{\mu_{l}}\right]<1$, and consequently matrix $I-\left(M_{l}^{-1} N_{l}\right)^{\mu_{l}}$ is nonsingular.

Now, as in the proof of Theorem 2 for weak nonnegative splittings of the first type, it is easy to see that the unique splitting $A=B_{l}-C_{l}$ induced by matrix $\left(M_{l}^{-1} N_{l}\right)^{\mu_{l}}$ such that $\left(M_{l}^{-1} N_{l}\right)^{\mu_{l}}=B_{l}^{-1} C_{l}$ is weak nonnegative of the first type. So, $\left\{\left(B_{l}, C_{l}, E_{l}\right)\right\}_{l=1}^{p}$ is a weak nonnegative multisplitting of the first type.

Now, by Lemmas 1 and 4(i) and equality (29) we have that

$$
\begin{aligned}
\sum_{l=1}^{p} E_{l} B_{l}^{-1} & =\sum_{l=1}^{p} E_{l}\left(I-\left(M_{l}^{-1} N_{l}\right)^{\mu_{l}}\right) A^{-1} \\
& =\sum_{l=1}^{p} E_{l}\left(\sum_{i=0}^{\mu_{l}-1}\left(M_{l}^{-1} N_{l}\right)^{i}\right) M_{l}^{-1} \\
& =R
\end{aligned}
$$

is a nonsingular matrix and $A=R^{-1}-\left(R^{-1}-A\right)$ is the unique weak nonnegative splitting of the first type induced by matrix $T$ such that $T=R\left(R^{-1}-A\right)$.

(ii) Similar to part (i) using a similar argument as in the proof of Theorem 2 for weak nonnegative splittings of the second type, using Remark 3 of Climent and Perea 6], and using part (ii)] instead of part (i)] of Lemma 4

(iii) Similar to part (i) using a similar argument as in the proof of Theorem 5 instead of Theorem 2, using Theorem 7.1.9 of Ortega 23] and using part(iii) instead of part (i) of Lemma 4.

Observe that condition " $A E_{l}=E_{l} A$ for $l=1,2, \ldots, p$ " in Lemma [4(ii) and Theorem 11][ii) is valid if " $E_{l}=\alpha_{l} I$ for $l=1,2, \ldots, p$ ".

\section{Parallel synchronous Alternating iterative methods}

With the aim of establishing more efficient parallel synchronous iterative methods, we introduce in this section two new methods that we call Model 1 and Model 2. These new methods combine Model A with the alternating iterative method introduced in Section 3. Also, we present convergence and comparison results for both methods.

Model 1: Let

$$
\left\{\left(M_{j, l}, N_{j, l}, E_{j, l}\right)\right\}_{l=1}^{p}, \quad j=1,2, \ldots, q,
$$


be $q$ multisplittings of $A$. We alternate $q$ global iterations of Model A obtained with each one of the $q$ multisplittings.

Model 2: Let

$$
\left\{\left(M_{j, l}, N_{j, l}, E_{l}\right)\right\}_{l=1}^{p} \quad j=1,2, \ldots, q,
$$

be $q$ multisplittings of $A$, each one with the same weighting matrices $\left\{E_{l}\right\}_{l=1}^{p}$. In each processor we apply the alternating iterative method (8) until a mutual phase time is reached when all processors are ready to contribute toward the global iteration, similar to Model A.

5.1. Convergence results for Model 1. First, we consider the mathematical formulation of Model 1. For the $q$ multisplittings (30) let

$$
\begin{aligned}
\boldsymbol{x}^{(k+j / q)}= & \sum_{l=1}^{p} E_{j, l}\left(M_{j, l}^{-1} N_{j, l}\right)^{\mu(j, k, l)} \boldsymbol{x}^{(k+(j-1) / q)} \\
& +\sum_{l=1}^{p} E_{j, l}\left(\sum_{i=0}^{\mu(j, k, l)-1}\left(M_{j, l}^{-1} N_{j, l}\right)^{i}\right) M_{j, l}^{-1} \boldsymbol{b}
\end{aligned}
$$

where $\mu(j, k, l)$ denotes the number of local iterations in the $l$ th processor at the $k$ th global iteration using the $j$ th multisplitting.

As in the sequential alternating iterative method of Section 3, we can eliminate vectors $\boldsymbol{x}^{(k+j / q)}$ for $j=1,2, \ldots, q-1$, obtaining the single iterative process

$$
\begin{aligned}
\boldsymbol{x}^{(k+1)} & =\prod_{j=1}^{q}\left(\sum_{l=1}^{p} E_{q-j+1, l}\left(M_{q-j+1, l}^{-1} N_{q-j+1, l}\right)^{\mu(q-j+1, k, l)}\right) \boldsymbol{x}^{(k)} \\
+ & \sum_{m=1}^{q}\left[\prod_{j=1}^{q-m}\left(\sum_{l=1}^{p} E_{q-j+1, l}\left(M_{q-j+1, l}^{-1} N_{q-j+1, l}\right)^{\mu(q-j+1, k, l)}\right)\right] \\
\times & {\left[\sum_{l=1}^{p} E_{m, l}\left(\sum_{i=0}^{\mu(m, k, l)-1}\left(M_{m, l}^{-1} N_{m, l}\right)^{i}\right) M_{m, l}^{-1}\right] \boldsymbol{b}, }
\end{aligned}
$$

for $k=0,1,2, \ldots$

where we assume that $\prod_{j=1}^{0}\left(\sum_{l=1}^{p} E_{q-j+1, l}\left(M_{q-j+1, l}^{-1} N_{q-j+1, l}\right)^{\mu(q-j+1, k, l)}\right)=I$, which is of the form (21) with

$$
T^{(k)}=\prod_{j=1}^{q}\left(\sum_{l=1}^{p} E_{q-j+1, l}\left(M_{q-j+1, l}^{-1} N_{q-j+1, l}\right)^{\mu(q-j+1, k, l)}\right) .
$$

Now, in a similar way to the sufficient convergence conditions for the nonstationary iterative method (22) introduced in the above section, we introduce sufficient convergence conditions for the nonstationary iterative process (32).

Theorem 12. Let $A$ be a nonsingular matrix. 
(i) Assume that $A$ is monotone and that the multisplittings (30) are weak nonnegative of the first type. If

$$
\mu(j, k, l) \geq 1, \quad j=1,2, \ldots, q, l=1,2, \ldots, p, k=1,2, \ldots,
$$

then the iterative method (32) converges to the unique solution of system (1) for any initial vector $\boldsymbol{x}^{(0)}$.

(ii) Assume that $A$ is monotone, that the multisplittings (30) are weak nonnegative of the second type, and that $E_{j, l}=\alpha_{j, l} I$ for $l=1,2, \ldots, p$ and $j=1,2, \ldots, q$. If (34) holds, then the iterative method (32) converges to the unique solution of system (1) for any initial vector $\boldsymbol{x}^{(0)}$.

(iii) Assume that $A$ is symmetric positive definite, that the multisplittings (30) are $P$-regular, and that $E_{j, l}=\alpha_{j, l} I$ for $l=1,2, \ldots, p$ and $j=1,2, \ldots, q$. If (34) holds, then the iterative method (32) converges to the unique solution of system (11) for any initial vector $\boldsymbol{x}^{(0)}$.

Proof. Each part follows by an argument similar to that in Theorems [6, [7] and 8] respectively.

Observe that, in a way similar to how we remark after Theorems 6] [7] and 8] assumption (34) can be weakened as follows:

$$
\mu(j, k, l) \geq 0, \quad l=1,2, \ldots, p, j=1,2, \ldots, q, k=0,1,2, \ldots,
$$

and infinitely many $k$ 's

$$
\mu(j, k, l) \geq 1, \quad l=1,2, \ldots, p, j=1,2, \ldots, q .
$$

On the other hand, in a way similar to as in Theorems 9] and 10, we can change the additional hypothesis " $E_{j, l}=\alpha_{j, l} I$ for $l=1,2, \ldots, p$ and $j=1,2, \ldots, q$ " of parts (ii) and (iii) of Theorem 12 by a condition on the number of local iterations $\mu(j, k, l)$. First we introduce the following result similar to Theorem 2.1 of Migallón, Penadés, and Szyld [19].

Theorem 13. Let $A$ be a nonsingular matrix and assume that each splitting of (30) is convergent; that is, $\rho\left(M_{j, l}^{-1} N_{j, l}\right)<1$ for $l=1,2, \ldots, p$ and $j=1,2, \ldots, q$. Given a fixed positive number $\theta<1$, let

$$
\eta_{j}=\frac{\theta}{\sum_{l=1}^{p}\left\|E_{j, l}\right\|}, \quad j=1,2, \ldots, q
$$

where $\|\cdot\|$ denotes any matrix norm such that $\|I\|=1$. Let $\tilde{\nu}_{j}$ be such that

$$
\left\|\left(M_{j, l}^{-1} N_{j, l}\right)^{\nu_{j}}\right\| \leq \eta_{j}
$$

for all $\nu_{j} \geq \tilde{\nu}_{j}, l=1,2, \ldots, p$ and $j=1,2, \ldots, q$. If the sequences of the number of iterations satisfy

$$
\mu_{j, k, l} \geq \tilde{\nu}_{j}
$$

for $j=1,2, \ldots, q, l=1,2, \ldots, p$, and $k=1,2, \ldots$, then the iterative method (32) converges to the unique solution of system (11) for any initial vector $\boldsymbol{x}^{(0)}$. 
Proof. From (33), (37), and (38), it follows that

$$
\begin{aligned}
\left\|T^{(k)}\right\| & \leq \prod_{j=1}^{q}\left(\sum_{l=1}^{p}\left\|E_{q-j+1, l}\right\|\left\|\left(M_{q-j+1, l}^{-1} N_{q-j+1, l}\right)^{\mu(q-j+1, k, l)}\right\|\right) \\
& \leq \prod_{j=1}^{q}\left(\sum_{l=1}^{p}\left\|E_{q-j+1, l}\right\| \eta_{j}\right) \\
& \leq \theta^{q}<\theta<1 .
\end{aligned}
$$

So $\lim _{k \rightarrow \infty}\left\|T^{(k)} T^{(k-1)} \cdots T^{(1)} T^{(0)}\right\|=0$, and consequently the iterative method (32) is convergent.

Now, taking into account that the weak nonnegative splittings of the second type of a monotone matrix are convergent and that the $p$-regular splittings of a symmetric positive definite matrix are also convergent, we obtain the following result similar to Theorems 9 and 10 .

Corollary 2. Let $A$ be a nonsingular matrix and consider a fixed positive number $\theta<1$.

(i) Assume that $A$ is monotone and that the multisplittings (30) are weak nonnegative of the second type. Let

$$
\eta_{j}=\frac{\theta}{\sum_{l=1}^{p}\left\|E_{j, l}\right\|}, \quad j=1,2, \ldots, q,
$$

where $\|\cdot\|$ denotes any matrix norm such that $\|I\|=1$. Let $\tilde{\nu}_{j}$ be such that

$$
\left\|\left(M_{j, l}^{-1} N_{j, l}\right)^{\nu_{j}}\right\| \leq \eta_{j}
$$

for all $\nu_{j} \geq \tilde{\nu}_{j}, l=1,2, \ldots, p$ and $j=1,2, \ldots, q$. If the sequences of the number of iterations satisfy inequality (39) for $j=1,2, \ldots, q, l=$ $1,2, \ldots, p$, and $k=1,2, \ldots$, then the iterative method (32) converges to the unique solution of system (1) for any initial vector $\boldsymbol{x}^{(0)}$.

(ii) Assume that $A$ is symmetric positive definite and that the multisplittings (30) are P-regular. Let

$$
\eta_{j}=\frac{\theta}{\sum_{l=1}^{p}\left\|E_{j, l}\right\|_{A}}, \quad j=1,2, \ldots, q .
$$

Let $\tilde{\nu}_{j}$ be such that

$$
\left\|\left(M_{j, l}^{-1} N_{j, l}\right)^{\nu_{j}}\right\|_{A} \leq \eta_{j}
$$

for all $\nu_{j} \geq \tilde{\nu}_{j}, l=1,2, \ldots, p$, and $j=1,2, \ldots, q$. If for $j=1,2, \ldots, q$ the sequences of the number of iterations satisfy inequality (39) for $j=$ $1,2, \ldots, q, l=1,2, \ldots, p$, and $k=1,2, \ldots$, then the iterative method (32) converges to the unique solution of system (1) for any initial vector $\boldsymbol{x}^{(0)}$.

If in the iterative process (32) we consider the particular case $\mu(j, k, l)=\mu(j, l)$, for $k=0,1,2, \ldots$, that is, the number of iterations on each multisplitting depends 
only of the multisplitting, then we obtain a stationary version of that method whose iteration matrix is

$$
T=\prod_{j=1}^{q}\left(\sum_{l=1}^{p} E_{q-j+1, l}\left(M_{q-j+1, l}^{-1} N_{q-j+1, l}\right)^{\mu(q-j+1, l)}\right) .
$$

Consider also the matrix

$$
\begin{aligned}
R= & \sum_{m=1}^{q}\left[\prod_{j=1}^{q-m}\left(\sum_{l=1}^{p} E_{q-j+1, l}\left(M_{q-j+1, l}^{-1} N_{q-j+1, l}\right)^{\mu(q-j+1, l)}\right)\right] \\
& \times\left[\sum_{l=1}^{p} E_{m, l}\left(\sum_{i=0}^{\mu(m, l)-1}\left(M_{m, l}^{-1} N_{m, l}\right)^{i}\right) M_{m, l}^{-1}\right]
\end{aligned}
$$

Theorem 14. Let $A$ be a nonsingular matrix. Consider the $q$ multisplittings defined by (30), assume that $\mu(j, k, l)=\mu(j, l) \geq 1$ for $k=0,1,2, \ldots$, and consider matrices $T$ and $R$ defined by (40) and (41), respectively.

(i) If $A$ is monotone and the $q$ multisplittings are weak nonnegative of the first type, then the stationary version of the iterative method (32) is convergent. Moreover, matrix $R$ is nonsingular and $A=R^{-1}-\left(R^{-1}-A\right)$ is the unique weak nonnegative splitting of the first type induced by matrix $T$ such that $T=R\left(R^{-1}-A\right)$.

(ii) If $A$ is monotone, the $q$ multisplittings are weak nonnegative of the second type, and we consider the additional hypothesis $E_{j, l}=\alpha_{j, l} I$, for $l=1,2, \ldots, p$ and $j=1,2, \ldots, q$, then the stationary version of the iterative method (32) is convergent. Moreover, matrix $R$ is nonsingular and $A=R^{-1}-\left(R^{-1}-A\right)$ is the unique weak nonnegative splitting of the second type induced by matrix $T$ such that $T=R\left(R^{-1}-A\right)$.

(iii) If $A$ is a symmetric positive definite matrix, the $q$ multisplittings are $P$ regular, and we consider the additional hypothesis $E_{j, l}=\alpha_{j, l} I$ for $l=$ $1,2, \ldots, p$ and $j=1,2, \ldots, q$, then the stationary version of the iterative method (32) is convergent. Moreover, matrix $R$ is nonsingular and $A=$ $R^{-1}-\left(R^{-1}-A\right)$ is the unique $P$-regular splitting induced by matrix $T$ such that $T=R\left(R^{-1}-A\right)$.

Proof. (i) By Theorem 12 the stationary version of the parallel iterative method (34) is convergent; that is, $\rho(T)<1$.

For $j=1,2, \ldots, q$, consider matrices

$$
\begin{aligned}
T_{j} & =\sum_{l=1}^{p} E_{j, l}\left(M_{j, l}^{-1} N_{j, l}\right)^{\mu(j, l)} \\
R_{j} & =\sum_{l=1}^{p}\left(\sum_{i=0}^{\mu(j, l)-1}\left(M_{j, l}^{-1} N_{j, l}\right)^{i}\right) M_{j, l}^{-1}
\end{aligned}
$$

defined by (28) and (29), respectively, for the multisplitting $\left\{M_{j, l}, N_{j, l}, E_{j, l}\right\}_{l=1}^{p}$.

By Theorem 11](i) $\rho\left(T_{j}\right)<1$, matrix $R_{j}$ is nonsingular, and $A=R_{j}^{-1}-\left(R_{j}^{-1}-A\right)$ is a weak nonnegative splitting of the first type induced by matrix $T_{j}$ such that $T_{j}=R_{j}\left(R_{j}^{-1}-A\right)$. 
Now, it is easy to see that

$$
T=\prod_{j=1}^{q} T_{q-j+1}, \quad R=\sum_{m=1}^{q}\left(\prod_{j=1}^{q-m} T_{q-j+1}\right) R_{m}, \quad \text { and } \quad R A=I-T .
$$

Clearly, $R$ is nonsingular because $\rho(T)<1$ and therefore $T=R\left(R^{-1}-A\right)$.

Finally, that $A=R^{-1}-\left(R^{-1}-A\right)$ is a weak nonnegative splittings of the first type follows from the above equalities and the fact that $A=R_{j}^{-1}-\left(R_{j}^{-1}-A\right)$ is the unique weak nonnegative splitting of the first type.

(ii) Similar to part (i) using Theorem[11|(ii) instead of Theorem 11|(i),

(iii) Similar to part (i) using Theorem 11](iii) instead of Theorem 11](i)

To finish this subsection, we introduce the following comparison result whose proof follows by Theorem 14 and Corollary 1.

Theorem 15. Let $A$ be a nonsingular and monotone matrix. Assume that the $q$ multisplittings defined by (30) are nonnegative and that $E_{j, l}=\alpha_{j, l} I$ for $l=$ $1,2, \ldots, p$ and $j=1,2, \ldots, q$. If $\mu(j, k, l)=\mu(j, l) \geq 1$ for $k=0,1,2, \ldots$, then

$$
\rho(T) \leq \min _{1 \leq j \leq q}\left\{\rho\left(T_{j}\right)\right\}
$$

where matrices $T$ and $T_{j}$ for $j=1,2, \ldots, q$ are defined by (40) and (42), respectively.

5.2. Convergence results for Model 2. We consider the mathematical formulation of Model 2.

For $l=1,2, \ldots, p$ we consider the iterative process (8) for the splitting $A=$ $M_{j, l}-N_{j, l}$ in (31):

$$
\boldsymbol{x}^{(k+1)}=T_{l}^{(k)} \boldsymbol{x}+R_{l}^{(k)} \boldsymbol{b}
$$

where

$$
T_{l}^{(k)}=\prod_{j=1}^{q}\left(M_{q-j+1, l}^{-1} N_{q-j+1, l}\right)^{\mu(q-j+1 k, l)}
$$

and

$$
R_{l}^{(k)}=\sum_{i=1}^{q}\left[\prod_{j=1}^{q-i}\left(M_{q-j+1, l}^{-1} N_{q-j+1, l}\right)^{\mu(q-j+1, k, l)}\right]\left[\sum_{m=0}^{\mu(i, k, l)-1}\left(M_{i, l}^{-1} N_{i, l}\right)^{m} M_{i, l}^{-1}\right] .
$$

Next we consider the parallel iterative method

$$
\boldsymbol{x}^{(k+1)}=\sum_{l=1}^{p} E_{l} T_{l}^{(k)} \boldsymbol{x}^{(k)}+\sum_{l=1}^{p} E_{l} R_{l}^{(k)} \boldsymbol{b}, \quad k=0,1,2, \ldots,
$$

which is of the form (2) with

$$
T^{(k)}=\sum_{l=1}^{p} E_{l} T_{l}^{(k)}, \quad k=0,1,2, \ldots,
$$

where matrices $T_{l}^{(k)}$ are defined by (44).

Now, as in the previous cases, we start with the convergence of the nonstationary iterative method (43) proving the following result.

Theorem 16. Let $A$ be a nonsingular matrix. 
(i) Assume that $A$ is monotone and that the $q$ multisplittings defined by (31) are weak nonnegative of the first type. If

$$
\mu(j, k, l) \geq 1, \quad j=1,2, \ldots, q, l=1,2, \ldots, p, k=0,1,2, \ldots,
$$

then the iterative method (43) converges for any initial vector $\boldsymbol{x}^{(0)}$ to the unique solution of system (11).

(ii) Assume that $A$ is monotone, that the $q$ multisplittings defined by (31) are weak nonnegative of the second type, and that $A E_{j, l}=E_{j, l} A$ for $l=$ $1,2, \ldots, p$ and $j=1,2, \ldots, q$. If (46) holds, then the iterative method (43) converges for any initial vector $\boldsymbol{x}^{(0)}$ to the unique solution of system (11).

(iii) Assume that $A$ is symmetric positive definite, that the $q$ multisplittings defined by (31) are P-regular, and that $E_{j, l}=\alpha_{j, l} I$ for $l=1,2, \ldots, p$ and $j=1,2, \ldots, q$. If (46) holds, then the iterative method (43) converges for any initial vector $\boldsymbol{x}^{(0)}$ to the unique solution of system (1).

Proof. (i) For a fixed $l$ with $1 \leq l \leq p$, as in the proofs of Theorem 1 for weak nonnegative splittings of the first type and Theorem 6 since $A^{-1} \geq 0$, there exists a vector $\boldsymbol{x}>0$ and a suitable constant $0 \leq \beta<1$ such that

$$
T_{l}^{(k)} \boldsymbol{x} \leq \beta \boldsymbol{x}, \quad k=0,1,2, \ldots,
$$

and by (45) we have that

$$
T^{(k)} \boldsymbol{x} \leq \beta \boldsymbol{x}, \quad k=0,1,2, \ldots
$$

So, $\lim _{k \rightarrow \infty}\left(T^{(k)} T^{(k-1)} \cdots T^{(1)} T^{(0)}\right)=0$ by Lemma 3 because $T^{(k)} \geq 0$ for $k=$ $0,1,2, \ldots$, and then the iterative process 43 is convergent.

(ii) As in part (i), similar to the proofs of Theorem 1 for weak nonnegative splittings of the second type and Theorem 7 .

(iii) As in part (i), similar to the proofs of Theorem 4 and Theorem 8

Here, as in Theorem 12, assumption (46) in Theorem 16 can be weakened by conditions (35) and (36).

On the other hand, in a way similar to as in previous sections, we can change the additional hypothesis " $A E_{j, l}=E_{j, l} A$ for $l=1,2, \ldots, p$ and $j=1,2, \ldots, q$ " and " $E_{j, l}=\alpha_{j, l} I$ for $l=1,2, \ldots, p$ and $j=1,2, \ldots, q$ " of parts (ii) and (iii) of Theorem [16] by a condition on the number of local iterations $\mu(j, k, l)$ in a way similar to as in Theorem 13 and Corollary 2 .

Theorem 17. Let $A$ be a nonsingular matrix and assume that each splitting of (44) is convergent; that is, $\rho\left(M_{j, l}^{-1} N_{j, l}\right)<1$ for $l=1,2, \ldots, p$ and $j=1,2, \ldots, q$. Given a fixed positive number $\theta<1$, let

$$
\eta=\left(\frac{\theta}{\sum_{l=1}^{p}\left\|E_{l}\right\|}\right)^{1 / q}
$$

where $\|\cdot\|$ denotes any matrix norm such that $\|I\|=1$. Let $\tilde{\nu}$ be such that

$$
\left\|\left(M_{j, l}^{-1} N_{j, l}\right)^{\nu}\right\| \leq \eta
$$


for all $\nu \geq \tilde{\nu}, l=1,2, \ldots, p$, and $j=1,2, \ldots, q$. If the sequences of the number of iterations satisfy

$$
\mu(j, k, l) \geq \tilde{\nu}
$$

for $j=1,2, \ldots, q, l=1,2, \ldots, p$, and $k=1,2, \ldots$, then the iterative method (45) converges to the unique solution of system (1) for any initial vector $\boldsymbol{x}^{(0)}$.

Corollary 3. Let $A$ be a nonsingular matrix and consider a fixed positive number $\theta<1$.

(i) Assume that $A$ is monotone and that the multisplittings (44) are weak nonnegative of the second type. Let

$$
\eta=\left(\frac{\theta}{\sum_{l=1}^{p}\left\|E_{l}\right\|}\right)^{1 / q},
$$

where $\|\cdot\|$ denotes any matrix norm such that $\|I\|=1$. Let $\tilde{\nu}$ be such that

$$
\left\|\left(M_{j, l}^{-1} N_{j, l}\right)^{\nu}\right\| \leq \eta
$$

for all $\nu \geq \tilde{\nu}, l=1,2, \ldots, p$, and $j=1,2, \ldots, q$. If the sequences of the number of iterations satisfy inequality (47) for $j=1,2, \ldots, q, l=$ $1,2, \ldots, p$, and $k=1,2, \ldots$, then the iterative method (45) converges to the unique solution of system (1) for any initial vector $\boldsymbol{x}^{(0)}$.

(ii) Assume that $A$ is symmetric positive definite and that the multisplittings (44) are P-regular. Let

$$
\eta=\left(\frac{\theta}{\sum_{l=1}^{p}\left\|E_{l}\right\|_{A}}\right)^{1 / q} .
$$

Let $\tilde{\nu}$ be such that

$$
\left\|\left(M_{j, l}^{-1} N_{j, l}\right)^{\nu}\right\|_{A} \leq \eta
$$

for all $\nu \geq \tilde{\nu}, l=1,2, \ldots, p$, and $j=1,2, \ldots, q$. If the sequences of the number of iterations satisfy inequality (47) for $j=1,2, \ldots, q, l=$ $1,2, \ldots, p$, and $k=1,2, \ldots$, then the iterative method (45) converges to the unique solution of system (11) for any initial vector $\boldsymbol{x}^{(0)}$.

As in the previous section, if in the iterative process (44) we consider the particular case $\mu(j, k, l)=\mu(j, l) \geq 1$ for $k=0,1,2, \ldots$, then we obtain a stationary version of that method, whose iteration matrix is

$$
\begin{aligned}
T & =\sum_{l=1}^{p} E_{l} T_{l} \\
& =\sum_{l=1}^{p} E_{l}\left[\prod_{j=1}^{q}\left(M_{q-j+1}^{-1} N_{q-j+1}\right)^{\mu(q-j+1, l)}\right] .
\end{aligned}
$$


Consider also matrix

$$
\begin{aligned}
R= & \sum_{l=1}^{p} E_{l} R_{l} \\
= & \sum_{l=1}^{p} E_{l}\left\{\sum_{i=1}^{q}\left[\prod_{j=1}^{q-i}\left(M_{q-j+1, l}^{-1} N_{q-j+1, l}\right)^{\mu(q-j+1, l)}\right]\right. \\
& \left.\times\left[\sum_{m=0}^{\mu(i, l)-1}\left(M_{i, l}^{-1} N_{i, l}\right)^{m} M_{i, l}^{-1}\right]\right\} .
\end{aligned}
$$

Then, with an argument similar to that in Theorem 14, we can establish the following result

Theorem 18. Let $A$ be a nonsingular matrix. Consider the $q$ multisplittings defined by (31), assume that $\mu(j, k, l)=\mu(j, l) \geq 1$ for $k=0,1,2, \ldots$, and consider matrices $T$ and $R$ defined by (48) and (49), respectively.

(i) If $A$ is monotone and the $q$ multisplittings are weak nonnegative of the first type, then the stationary version of the iterative method (43) is convergent. Moreover, matrix $R$ is nonsingular and $A=R^{-1}-\left(R^{-1}-A\right)$ is the unique weak nonnegative splitting of the first type induced by matrix $T$ such that $T=R\left(R^{-1}-A\right)$.

(ii) If $A$ is monotone, the $q$ multisplittings are weak nonnegative of the second type, and we consider the additional hypothesis $A E_{l}=E_{l} A$, for $l=1,2, \ldots, p$, then the stationary version of the iterative method (43) is convergent. Moreover, matrix $R$ is nonsingular and $A=R^{-1}-\left(R^{-1}-A\right)$ is the unique weak nonnegative splitting of the second type induced by matrix $T$ such that $T=R\left(R^{-1}-A\right)$.

(iii) If $A$ is a symmetric positive definite matrix, the $q$ multisplittings are $P$ regular, and we consider the additional hypothesis $E_{l}=\alpha_{l} I$ for $l=1$, $2, \ldots, p$, then the stationary version of the iterative method (43) is convergent. Moreover, matrix $R$ is nonsingular and $A=R^{-1}-\left(R^{-1}-A\right)$ is the unique $P$-regular splitting induced by matrix $T$ such that $T=R\left(R^{-1}-A\right)$.

To finish this section, we establish an upper bound for the spectral radius of matrix $T$ defined by (48).

Theorem 19. Let $A$ be a nonsingular and monotone matrix. Assume that the $q$ splittings defined by (31) are weak nonnegative of the first type and that $\mu(j, k, l)=$ $\mu(j, l)$ for $k=0,1,2, \ldots$. Consider also a weak nonnegative splitting of the second type $A=M-N$, such that

$$
M_{j, l} \leq M, \quad l=1,2, \ldots, p, j=1,2, \ldots, q .
$$

Then

$$
\rho(T) \leq \rho\left(M^{-1} N\right)<1
$$

where $T$ is the matrix defined by (48).

Proof. Clearly $\rho\left(M^{-1} N\right)<1$. 
Let

$$
T_{l}=\prod_{j=1}^{q}\left(M_{q-j+1, l}^{-1} N_{q-j+1, l}\right)^{\mu(q-j+1, l)}, \quad l=1,2, \ldots, p .
$$

By Theorem 2, $\rho\left(T_{l}\right)<1$ and the unique splitting $A=B_{l}-C_{l}$ induced by matrix $T_{l}$ such that $T_{l}=B_{l}^{-1} C_{l}$ is weak nonnegative of the first type. Consequently $\left\{\left(B_{l}, C_{l}, E_{l}\right)\right\}_{l=1}^{p}$ is a weak nonnegative multisplitting of the first type, and by Theorem 1.a of O'Leary and White [22],

$$
\rho(T)=\rho\left(\sum_{l=1}^{p} B_{l}^{-1} C_{l}\right)<1 .
$$

Now, by Lemma1 (see also (14) and (50)) we obtain that

$$
B_{l}^{-1} \geq M_{j, l}^{-1} \geq M^{-1}, \quad l=1,2, \ldots, p, j=1,2, \ldots, q ;
$$

that is, $B_{l} \leq M$ for $l=1,2, \ldots, p$, and by Theorem 3.3 of Climent and Perea [7, inequality (51) follows.

\section{Numerical RESUlts}

In this section we present numerical results on Models A, 1 and 2 introduced previously. We have run our experiments, coded in Fortran using BSPlib [14, in an IBM SP2 computer. To implement these methods, we have considered the Laplace equation satisfying Dirichlet boundary conditions in the rectangle $\Omega=$ $[0, a] \times[0, a]$. We discretized the domain $\Omega$ with $\sqrt{n} \times \sqrt{n}$ points equally spaced. This discretization yields the linear system (1) where $A=\operatorname{tridiag}[-I, C,-I]$ is a $\sqrt{n} \times \sqrt{n}$ block tridiagonal matrix, $I$ is the $\sqrt{n} \times \sqrt{n}$ identity and $C=\operatorname{tridiag}[-1,4,-1]$ is a $\sqrt{n} \times \sqrt{n}$ tridiagonal matrix.

In order to define the splittings $A=M_{l}-N_{l}$, for $l=1,2, \ldots, p$, we consider matrix $A$ as

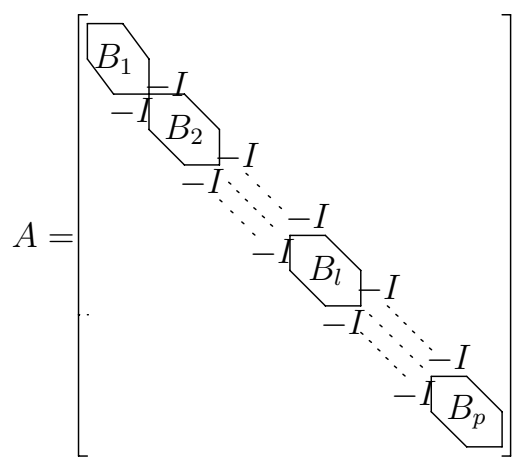

where $B_{l}=\operatorname{tridiag}[-I, C,-I]$, for $l=1,2, \ldots, p$. In all three models we consider that each processor $P_{l}$, for $l=1,2, \ldots, p$, makes a fixed number of local iterations $\mu(j, k)$ for the $j$ th splitting at the $k$ th global iteration. The stopping criterion used was

$$
\sum_{i=1}^{n}\left|x_{i}^{(k+1)}-x_{i}^{(k)}\right|<10^{-12}
$$


For Model A we consider the multisplitting (21) defined by

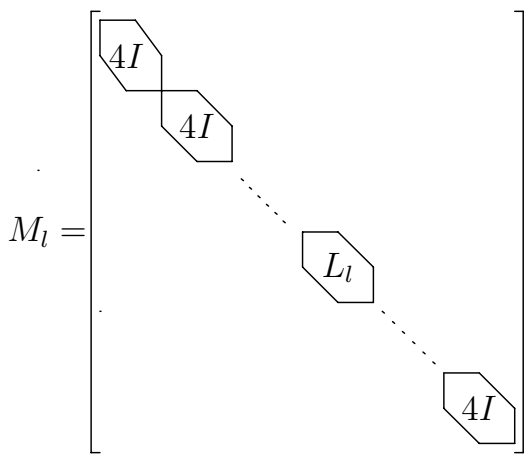

and $N_{l}=M_{l}-A$ where $L_{l}$ is the lower triangular part of $B_{l}$. Matrix $E_{l}$ is partitioned accordingly with $M_{l}$ changing $L_{l}$ by $I$ and $4 I$ by the zero matrix.

For Model 1 we consider $q=p$ and the multisplittings (30) defined by

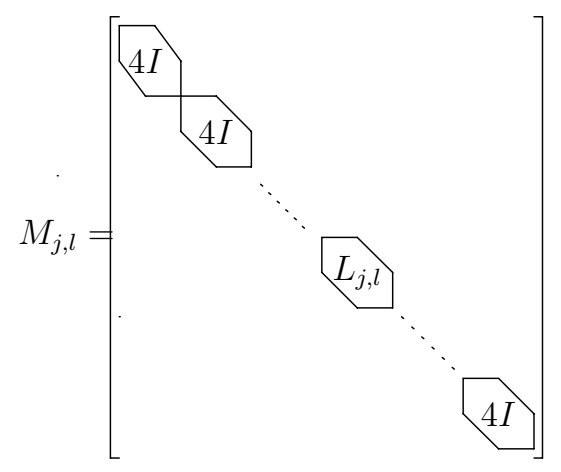

and $N_{j, l}=M_{j, l}-A$ where $L_{j, l}$ is the lower triangular part of

$$
B_{(j+l-1) \bmod p} .
$$

Matrix $E_{j, l}$ is partitioned accordingly with $M_{j, l}$ changing $L_{j, l}$ by $I$ and $4 I$ by the zero matrix.

Finally, for Model 2 we consider the multisplittings (31) as in the previous case, but matrix $E_{l}$ is defined as in Model A.

Experiments were performed with different sizes of matrices and different values for $\mu(j, k)$, obtaining analogous results for all cases. We find that Model 1 needs fewer iterations than the other ones. Table 1 shows the number of global iterations for Models A, 1 and 2 as a function of the matrix size.

Figures [1] and 2] show the results obtained for $n=1024$ and $p=2$ and for $n=4096$ and $p=4$, respectively. 


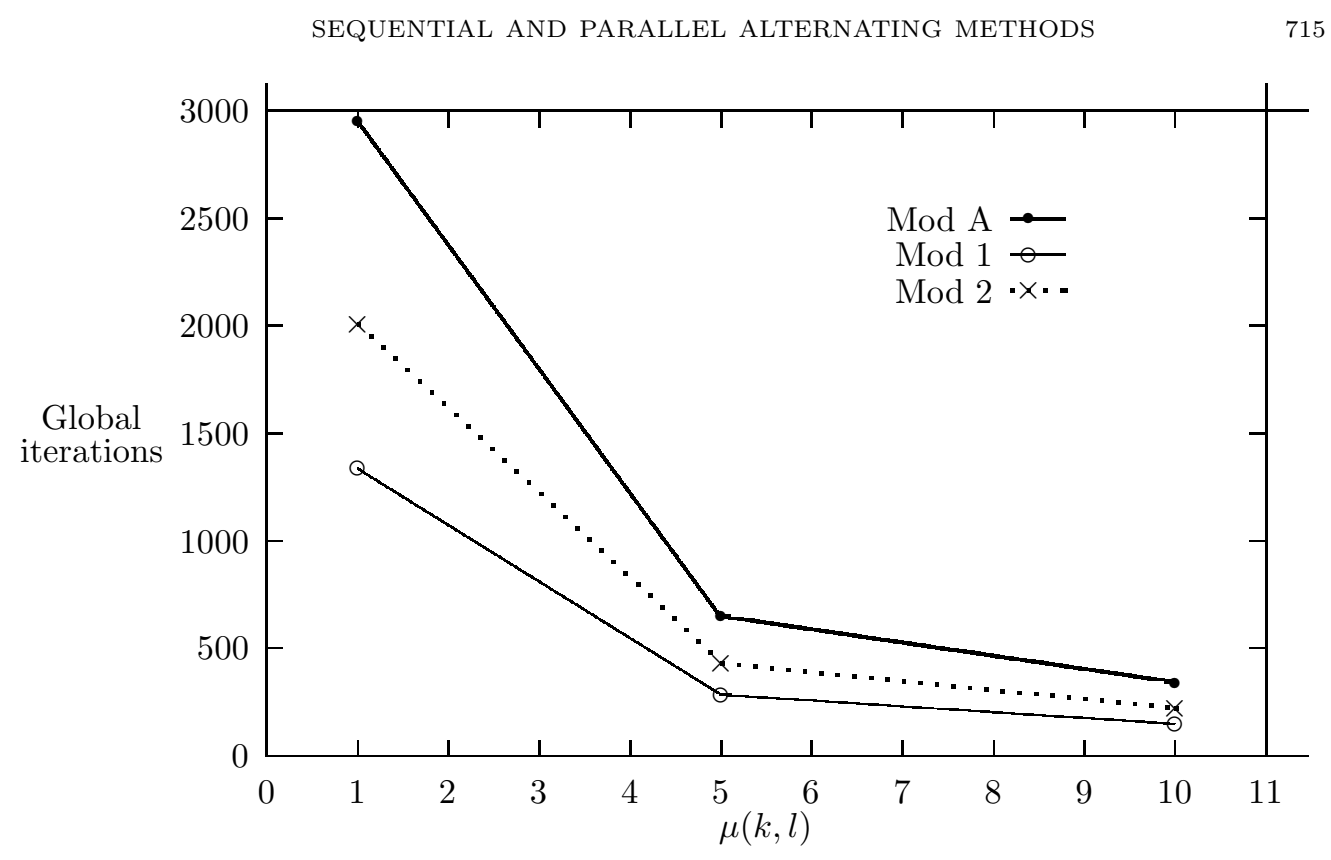

Figure 1. Global iterations for $n=1024$ and $p=2$.

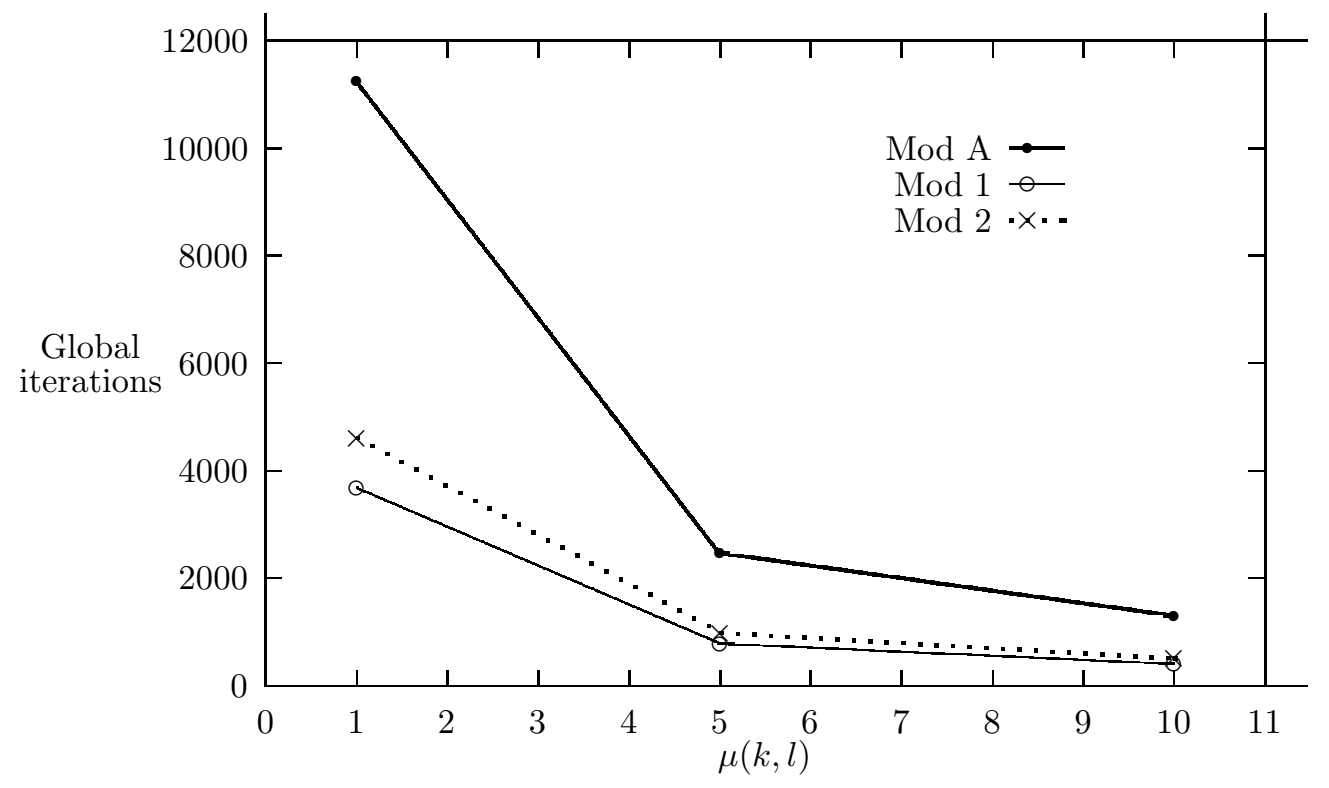

Figure 2. Global iterations for $n=4096$ and $p=4$. 
TABLE 1. Global iterations for models A, 1 and 2.

\begin{tabular}{|c|c|ccc|ccc|}
\cline { 3 - 8 } \multicolumn{2}{c|}{} & \multicolumn{3}{c|}{1024} & \multicolumn{3}{c|}{ Mo96el } \\
\cline { 3 - 8 } \multicolumn{2}{c|}{} & \multicolumn{3}{c|}{ Model } & \\
\hline$p$ & $\mu(j, k)$ & A & 1 & 2 & $A$ & 1 & 2 \\
\hline \multirow{4}{*}{2} & 1 & 2957 & 1338 & 2005 & 11054 & 5024 & 7489 \\
& 5 & 649 & 286 & 431 & 2390 & 1070 & 1602 \\
& 10 & 340 & 148 & 224 & 1239 & 550 & 826 \\
\hline \multirow{4}{*}{4} & 1 & 3057 & 983 & 1240 & 11244 & 3684 & 4610 \\
& 5 & 690 & 210 & 268 & 2468 & 784 & 987 \\
& 10 & 368 & 109 & 139 & 1293 & 403 & 510 \\
\hline \multirow{4}{*}{8} & 1 & 3235 & 620 & 710 & 11581 & 2322 & 2631 \\
& 5 & 768 & 133 & 152 & 2616 & 494 & 564 \\
& 10 & 421 & 69 & 78 & 1395 & 254 & 291 \\
\hline
\end{tabular}

\section{REFERENCES}

[1] A. Berman and R.J. Plemmons. Nonnegative Matrices in the Mathematical Sciences. Academic Press, New York, 1979. Reprinted by SIAM. Philadelphia, PA, 1994. MR 95e:15013

[2] M. BEnZi AND D.B. SzYlD. Existence and uniqueness of splittings for stationary iterative methods with applications to alternating methods. Numerische Mathematik, 76: 39321 (1997). MR 98c:65041

[3] R. Bru, L. Elsner, and M. Neumann. Models of parallel chaotic iteration methods. Linear Algebra and its Applications, 103: 175-192 (1988). MR 90b:65255

[4] R. Bru, L. Elsner, and M. Neumann. Convergence of infinite products of matrices and inner-outer iteration schemes. Electronic Transactions on Numerical Analysis, 2: 183193 (1994). MR 95i:65046

[5] R. Bru, V. Migallón, and J. Penadés. Chaotic methods for the parallel solution of linear systems. Computing Systems in Engineering, 6: 385-390 (1995).

[6] J.-J. Climent and C. Perea. Some comparison theorems for weak nonnegative splittings of bounded operators. Linear Algebra and its Applications, 275/276: 77-106 (1998). MR 99j:65043

[7] J.-J. Climent and C. Perea. Convergence and comparison theorems for multisplittings. Numerical Linear Algebra with Applications, 6: 93-107 (1999). MR 2000c:65023

[8] V. Conrad and Y. Wallach. Alternating methods for sets of linear equations. Numerische Mathematik, 32: 105-108 (1979). MR 80b:65042

[9] G. Csordas and R. VArga. Comparisons of regular splittings of matrices. Numerische Mathematik, 44: 23-35 (1984). MR 85g:65043

[10] L. Elsner. Comparisons of weak regular splittings and multisplitting methods. Numerische Mathematik, 56: 283-289 (1989). MR 85g:65043

[11] A. Frommer and G. Mayer. Convergence of relaxed parallel multisplitting methods. Linear Algebra and its Applications, 119: 141-152 (1989). MR 90f:65049

[12] A. Frommer And G. MaYer. On the theory and practice of multisplitting methods in parallel computation. Computing, 49: 63-74 (1992). MR 93e:65158

[13] A. Frommer and D.B. Szyld. Weighted max norms, splittings, and overlapping additive Schwarz iterations. Numerische Mathematik, 83: 259-278 (1999). MR 2000g:65023

[14] J.M.D. Hill, B. McColl, D.C. Stefanescu, M.W. Goudreau, K. Lang, S.B. Rao, T. Suel, T. Tsantilas, and R.H. Bisseling. BSPlib: The BSP Programming Library. Parallel Computing, 24: 1947-1980 (1998).

[15] P.J. Lanzkron, D.J. Rose, and D.B. Szyld. Convergence of nested classical iterative methods for linear systems. Numerische Mathematik, 58: 685-702 (1991). MR 92e:65045

[16] G.I. MARChuK. Splitting and alternating direction methods. In P.G. CiarlET AND J.L. Lions (editors). Handbook of Numerical Analysis, Vol. I, pages 197-462. North Holland, New York, NY, 1990. 
[17] I. Marek And D.B. Szyld. Comparison theorems for weak splittings of bounded operators. Numerische Mathematik, 58: 389-397 (1990). MR 92f:65070

[18] V.A. Miller And M. Neumann. A note on comparison theorems for nonnegative matrices. Numerische Mathematik, 47: 427-434 (1985). MR 87a:65062

[19] V. Migallón, J. Penadés, And D.B. Szyld. Nonstationary multisplittings with general weighting matrices. SIAM Journal on Matrix Analysis and Applications, 22: 1089-1094 (2001). MR 2001m:65046

[20] R. NAbBen. A note on comparison theorems for splittings and multisplittings of Hermitian positive definite matrices. Linear Algebra and its Applications, 233: 67-80 (1996). MR 97a:15035

[21] M. Neumann and R.J. Plemmons. Convergence of parallel multisplitting iterative methods for $M$-matrices. Linear Algebra and its Applications, 88/89: 559-573 (1987). MR 88k:65143

[22] D.P. O'Leary and R.E. White. Multi-splittings of matrices and parallel solution of linear systems. SIAM Journal on Algebraic and Discrete Methods, 6: 630-640 (1985). MR 86h:65047

[23] J.M. Ortega. Numerical Analysis, A second course. Academic Press, New York, NY, 1972. Reprinted by SIAM, Philadelphia, PA, 1992. MR 90k:65005

[24] R.S. Varga. Matrix Iterative Analysis. Prentice-Hall, Englewood Cliffs, NJ, 1962. MR 28:1725

[25] D. WAng. On the convergence of the parallel multisplitting AOR algorithm. Linear Algebra and its Applications, 154/156: 473-486 (1991). MR 92h:65210

[26] Z.I. WoźNICKI. Nonnegative splitting theory. Japan Journal on Industrial and Applied Mathematics, 11: 289-342 (1994). MR 95g:65051

[27] D.M. Young. Iterative Solution of Large Linear Systems. Academic Press, New York, NY, 1971. MR 46:4698

Departament de Ciència de la Computació i Intel-ligència Artificial, Universitat D'Alacant, Ap. Correus 99, E-03080 Alacant, Spain

E-mail address: jcliment@dccia.ua.es

Departamento de Estadística y Matemática Aplicada, Universidad Miguel Hernández, Escuela Politécnica Superior de Orihuela, E-03550, Orihuela, Spain

E-mail address: perea@umh.es

Departament de Ciència de la Computació i Intel-ligència Artificial, Universitat D'Alacant, Ap. Correus 99, E-03080 Alacant, Spain

E-mail address: tortosa@dccia.ua.es

Departament de Ciència de la Computació i Intel-ligència Artificial, Universitat D'Alacant, Ap. Correus 99, E-03080 Alacant, Spain

E-mail address: zamora@dccia.ua.es 